
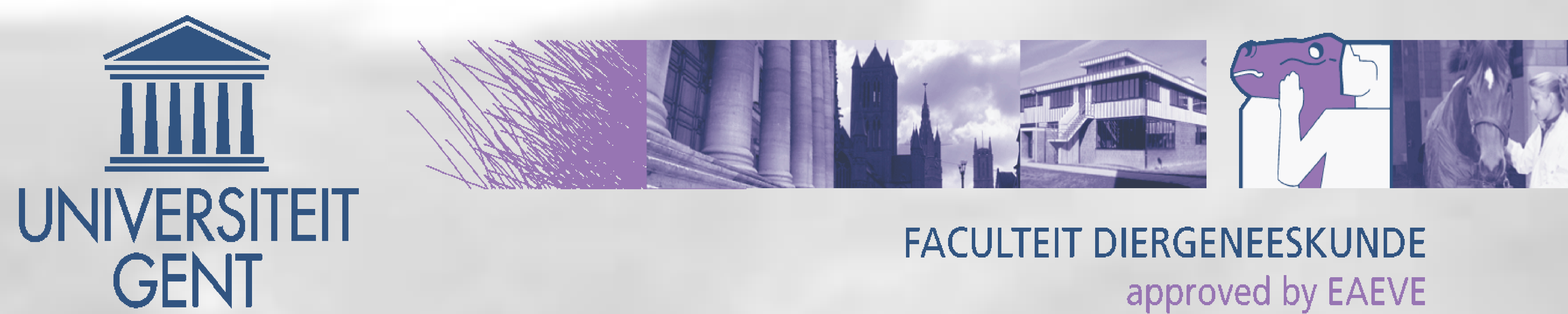

FACULTEIT DIERGENEESKUNDE

approved by EAEVE

\title{
Bony fusion after reimplantation of removed autogenous cancellous bone in a dog treated surgically for disk associated wobbler syndrome by a distractable vertebral titanium cage.
}

\author{
Department of Pathology, Bacteriology and avian diseases \\ Faculty of Veterinary Medicine \\ University of Ghent \\ Belgium \\ Beatrice.Wegge@ugent.be
}

Beatrice Wegge, Steven De Decker, Jacques Caemaert, Ingrid Gielen, Luc Van Ham and Koen Chiers.

Introduction: Disk associated wobbler syndrome (DAWS) is the most common cause of cervical spondylomyelopathy in dogs. DAWS is generally treated by surgery. In this case we evaluated the use of a distractable titanium cage for surgical treatment in dogs.

Materials and methods: A 10-year-old Dalmatian with DAWS was submitted for surgical treatment. After total discectomy of $\mathrm{C} 5-\mathrm{C} 6$ and $\mathrm{C} 6-\mathrm{C} 7$, an median corpectomy of $\mathrm{C} 6$ was performed by a pneumatic drill. The removed cancellous bone was carefully collected to act as an autogenous cancellous bone graft. Subsequently, the titanium implant was placed and affixed in the defect. Finally it was filled and covered with the collected cancellous bone.The dog improved and survived for 22 months and was euthanised because of lumbosacral stenosis. The intervertebral tissue surrounding the implant was removed and submitted for histopathological examination to confirm a bony fusion of the bone graft with the lateral edges of the vertebral body.

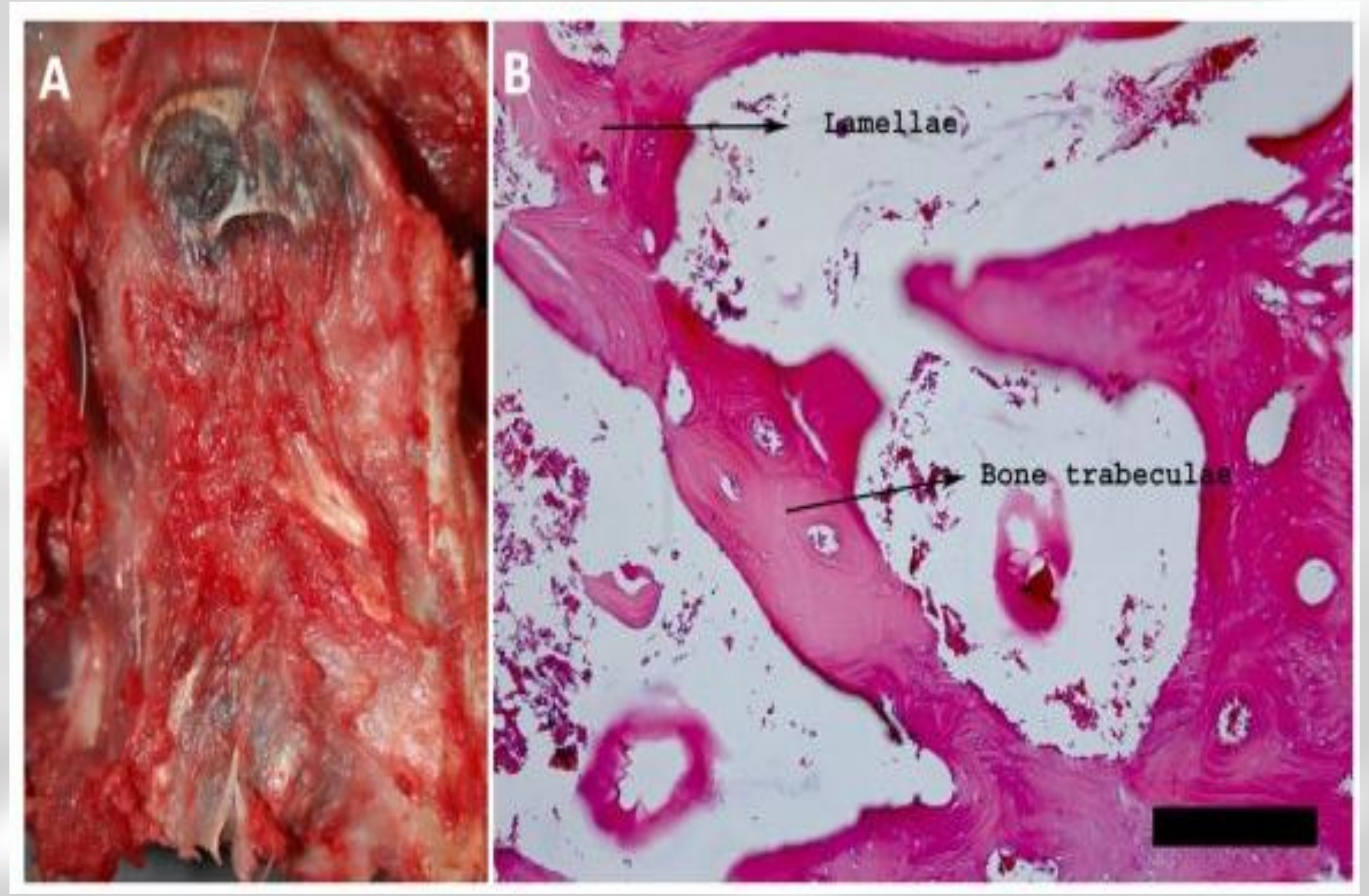

Figure 2. Pathological (A) and histopathological (B) examination of autogenous cancellous bone graft surrounding the implant (A) The incorporation of the titanium cage into the vertebral body. (B) HE staining of a trabeculum composed of cancellous bone.

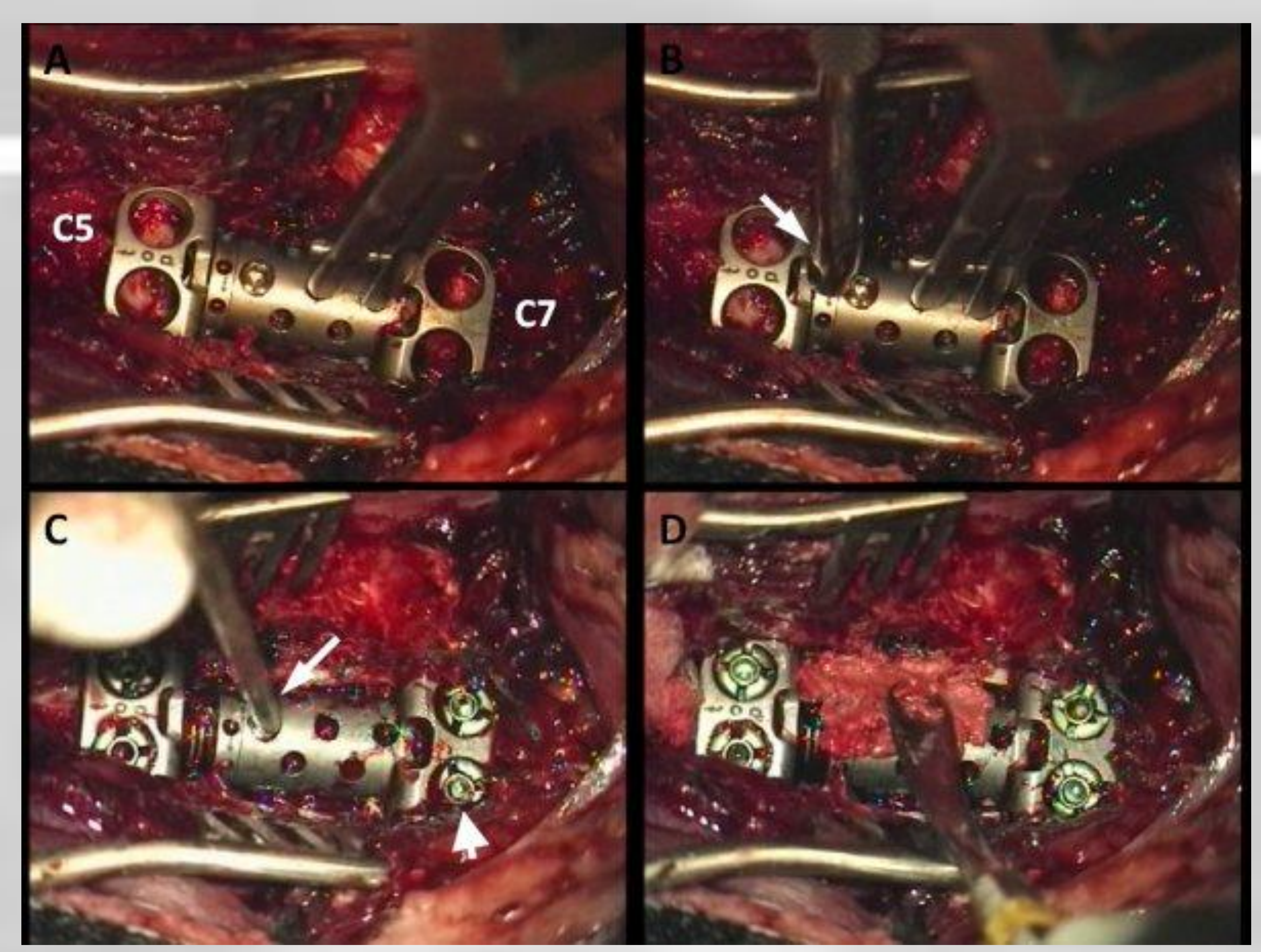

Figure 1. Intraoperative pictures of dog 1. (A) After the median corpectomy was created, the titanium cage was placed in the bony defect of C6. (B) Distraction of the implant by a mechanism inherent to the implant design (arrow). (C) Placement of set screw to maintain distraction (arrow). Fixation of the distractable cage by self-expandable monocortical screws (arrowhead). (D) Cage was filled and covered by autogenous cancellous bone graft

Results: Samples of the lateral and ventral sides showed mature cancellous bone. The spinal cord section at the level of the implant showed no signs of compression.

Conclusion: This is the first veterinary report demonstrating bony fusion after reimplantation of mechanically removed cancellous bone after a vertebral corpectomy. 